\title{
Bi-Directional Fast Charging Study Report
}

February 2012
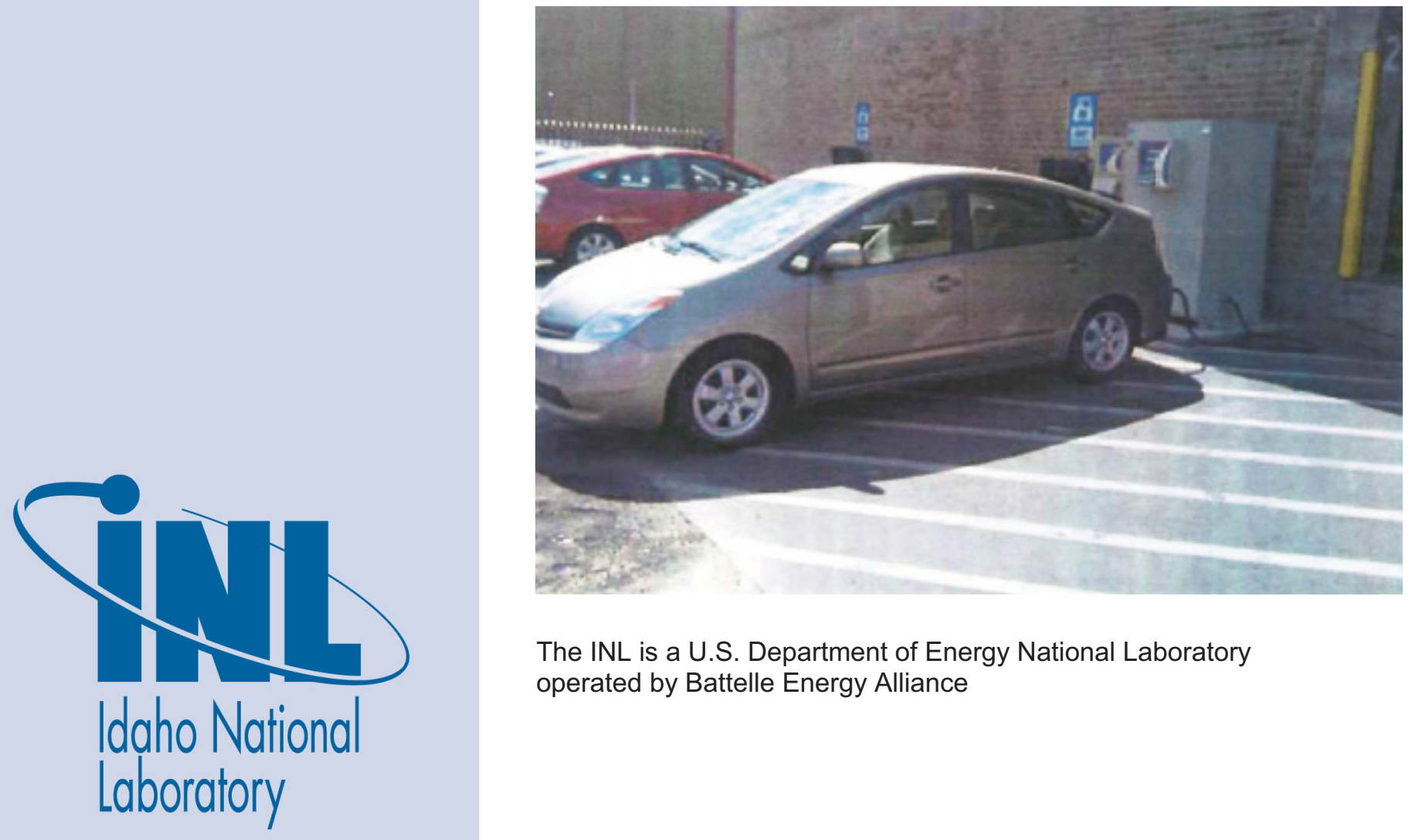

The INL is a U.S. Department of Energy National Laboratory operated by Battelle Energy Alliance 


\section{DISCLAIMER}

This information was prepared as an account of work sponsored by an agency of the U.S. Government. Neither the U.S. Government nor any agency thereof, nor any of their employees, makes any warranty, expressed or implied, or assumes any legal liability or responsibility for the accuracy, completeness, or usefulness, of any information, apparatus, product, or process disclosed, or represents that its use would not infringe privately owned rights. References herein to any specific commercial product, process, or service by trade name, trade mark, manufacturer, or otherwise, does not necessarily constitute or imply its endorsement, recommendation, or favoring by the U.S. Government or any agency thereof. The views and opinions of authors expressed herein do not necessarily state or reflect those of the U.S. Government or any agency thereof. 


\title{
Bi-Directional Fast Charging Study Report
}

\author{
Tyler Gray, ECOtality North America
}

James Francfort, Idaho National Laboratory

February 2012

Idaho National Laboratory Idaho Falls, Idaho 83415

\author{
http://www.inl.gov
}

Prepared for the

U.S. Department of Energy

Office of Nuclear Energy

Under DOE Idaho Operations Office

Contract DE-AC07-05ID14517 


\section{CONTENTS}

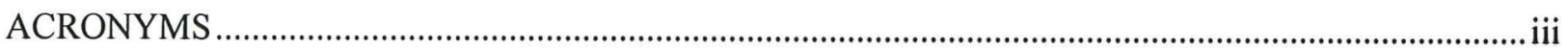

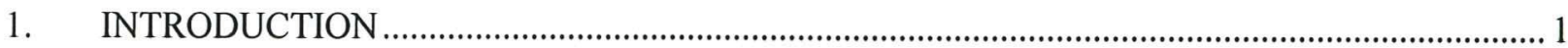

2. PLUG-IN HYBRID ELECTRIC VEHICLE FAST CHARGE CONVERSION ............................... 1

3. HARDWARE INFRASTRUCTURE REQUIREMENTS ......................................................... 3

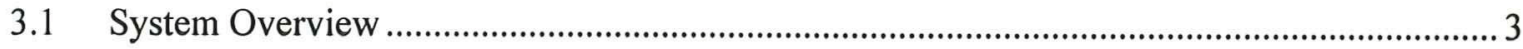

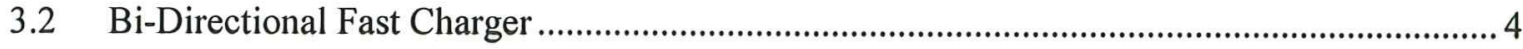

3.3 GridPoint Vehicle Connectivity Modules ........................................................................ 4

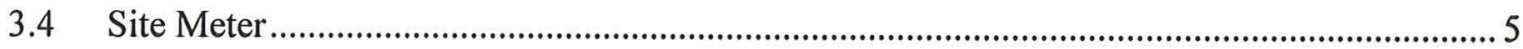

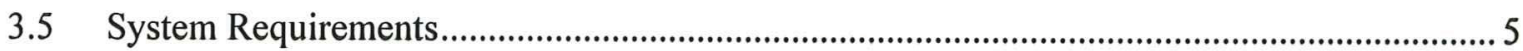

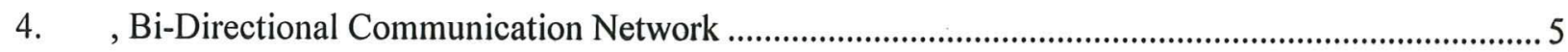

4.1 Hardware-to-Hardware Communication.......................................................................

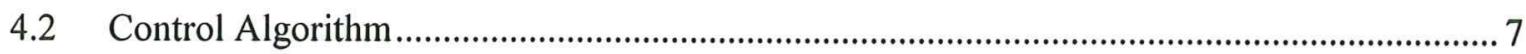

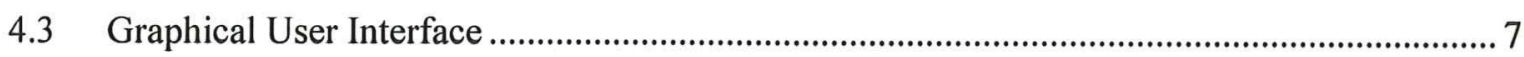

4.4 Web Application Programming Interface ....................................................................... 7

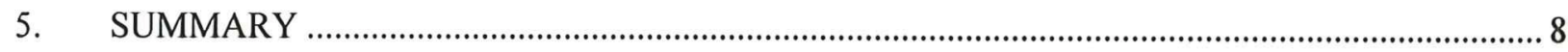

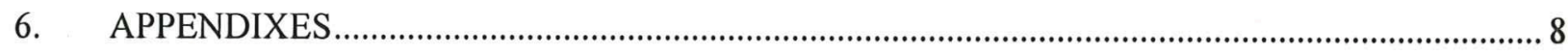

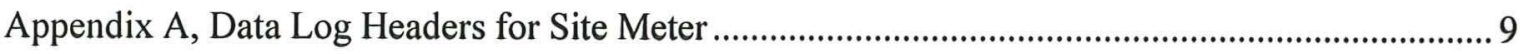

Appendix B, Web Application Programming Interface Calls ..................................................... 10

\section{FIGURES}

1. EV100 connector and standard 120-VAC connector that is mounted onto the Prius back bumper..... 2

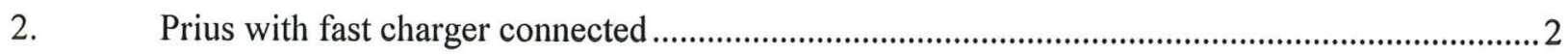

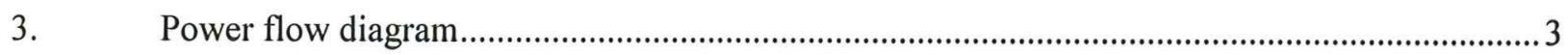

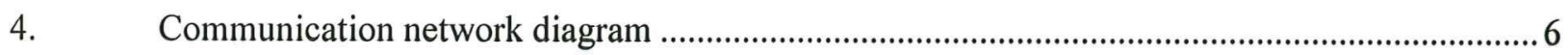

5. Graphical user interface with variable settings window ........................................................ 8 


\section{ACRONYMS}

$\mathrm{A} / \mathrm{C} \quad$ air conditioner

API application programming interface

CAN controller area network

CCC charge controller card

GUI graphical user interface

VCM vehicle connectivity module 


\section{Bi-Directional Fast Charging Study Report}

\section{INTRODUCTION}

This report details the hardware and software infrastructure needed to demonstrate the possibility of utilizing battery power in plug-in hybrid electric vehicles and electric vehicles with a bi-directional fast charger to support/offset peak building loads. This document fulfills deliverable requirements for ECOtality North America's support to the Idaho National Laboratory.

\section{PLUG-IN HYBRID ELECTRIC VEHICLE FAST CHARGE CONVERSION}

This demonstration took place before plug-in vehicles were available to the general public from traditional large-scale vehicle manufacturers. Therefore, an aftermarket conversion by A123/Hymotion of a Toyota Prius (VIN: JTDKB20U740012721) from a hybrid electric vehicle to a plug-in hybrid electric vehicle was used. This conversion included adding A123/Hymotion's L5 plug-in conversion modules, which contain a 4.7-kWh Nanophosphate lithium-ion battery pack. The L5 battery pack is connected in parallel with the stock Prius nickel-metal-hydride battery pack, which extends the electric drive capabilities and increases the fuel economy. The L5 battery pack is designed to be recharged with a standard $120 \mathrm{VAC}$ outlet at Level 1 power levels. At this power level, the charge time from fully discharged is specified to be approximately 5.5 hours.

For this demonstration, the A123/Hymotion battery pack needed to be configured with hardware to make it fast-charge ${ }^{\text {a }}$ capable. To accomplish this, power cables were added directly to the positive and negative terminals of the battery pack. These cables extend from the pack enclosure and into a National Electrical Manufacturers Association-certified electric enclosure that contains a contact for each connection. Power out of the contacts goes to the power pins of an EV100 power connector. The EV100 power connector contains three 100-A power pins and six auxiliary signal pins and is mounted on the back bumper of the Prius (Figures 1 and 2). The contactors for battery charge/discharge power are controlled by an isolated $12-\mathrm{V}$ signal wire that provides input from two of the auxiliary pins, one for $+12 \mathrm{~V}$ and one for reference ground, on the EV100. Of the other four auxiliary pins on the EV100, two are used for controller-area network (CAN) message communications between the L5 battery pack and the rest of the system, and two are used for a pulse width-modulated interconnect signal. The pulse width-modulated interconnect signal is used by the fast charger as a loop-back signal to indicate that the connector is plugged in. In the vehicle, this signal is used to ensure no charging takes place when the vehicle is turned on. This is done by placing another contact in series with the interconnect signal, opening the line with any key-on event stopping power flow, and disconnecting the $12-\mathrm{V}$ signal.

\footnotetext{
a The term 'fast charge' refers to the $1 \mathrm{C}$ or greater charge rate. For the $\mathrm{A} 123 /$ Hymotion battery, this corresponds to $25.3 \mathrm{~A}$ or greater.
} 


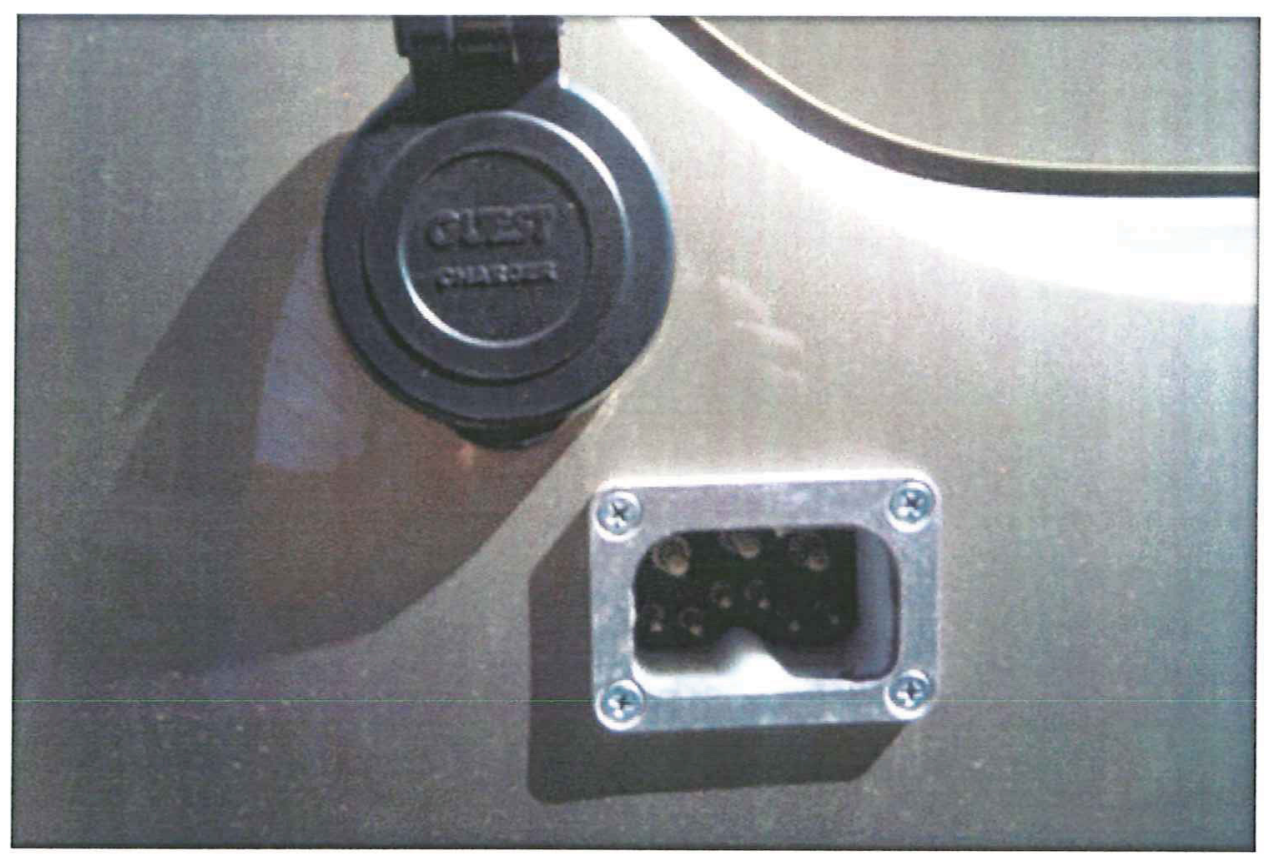

Figure 1. EV100 connector and standard 120-VAC connector that is mounted onto the Prius back bumper.

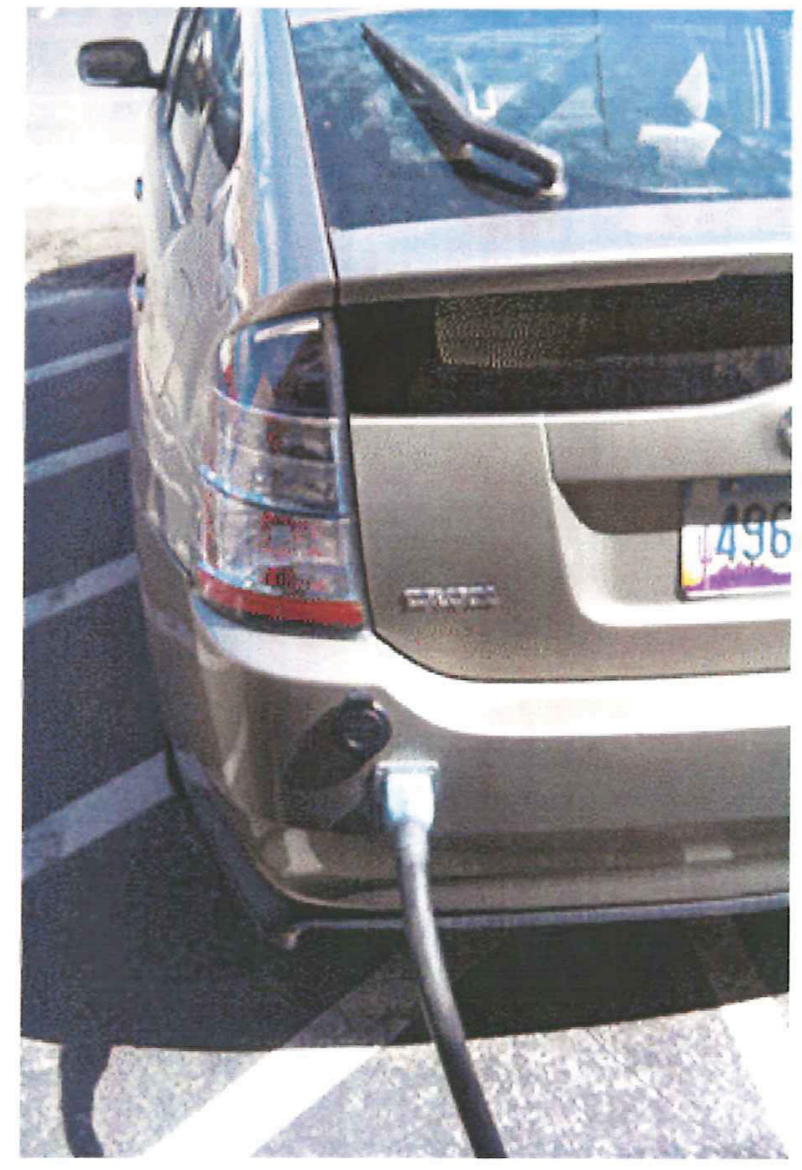

Figure 2. Prius with fast charger connected. 


\section{HARDWARE INFRASTRUCTURE REQUIREMENTS}

\subsection{System Overview}

For this task, a system was developed for monitoring and controlling bi-directional vehicle fast-charging events and how these charge events relate to site power. This system contains a bi-directional fast charger with charger controller, a fast charger vehicle connectivity module (VCM) made by GridPoint, a site power meter, an A/C VCM, and a vehicle VCM. The function of each component within the system is described in the following subsections. A graphical overview of the entire system is presented in Figure 3.

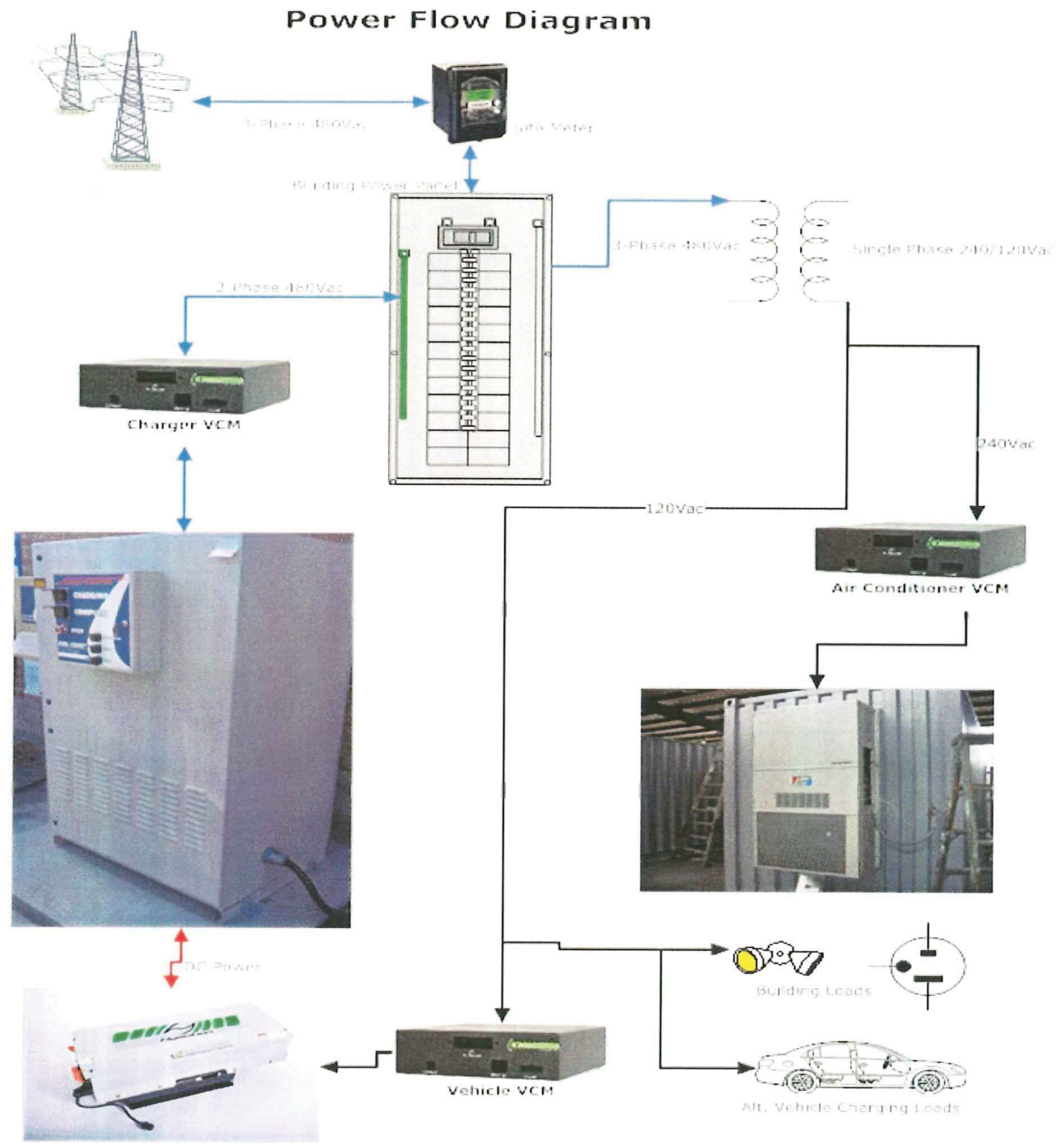

Figure 3. Power flow diagram. 


\subsection{Bi-Directional Fast Charger}

To meet the needs of this project, the fast charger had to be capable of charging the L5 pack from the electric grid, as well as discharging it back onto the grid. Typically, this bi-directional capability presents a problem, because commercial fast charge-capable hardware at the power levels desired is difficult to find at the time of the experiment and is typically expensive. An ECOtality North America charger with this bi-directional function (i.e., the Minit-Charger ${ }^{\mathrm{TM}} 25 \mathrm{AFE}$ ) was used. The $25 \mathrm{AFE}$ is capable of more than $25 \mathrm{~kW}$ of charge and discharge power, with a current maximum of $400 \mathrm{~A}$ and a voltage maximum of $400 \mathrm{~V}$. This charger normally is manually controlled by an analog signal that is proportional to the current magnitude and direction of the charger and also by digital signals that control power contactors. To allow the charger to run autonomously and communicate with other hardware, a charger controller card (CCC) (also made by ECOtality North America) was used.

The CCC is a programmable printed circuit board used to control industrial chargers. The CCC for this project features the following:

- RS232 communication port for use in software design debugging

- CAN communication port, used for communicating power magnitude and direction with the fast charger VCMs and system parameters such as maximum and minimum cell voltage and temperature with the L5 battery management system

- Digital input and output lines used to monitor the state of the 25 AFE charge cable with respect to the vehicle and the charge contacts

- Relay drivers used to open and close various $24-\mathrm{V}$ relays

- Analog-to-digital inputs used for voltage and current monitoring

- Digital-to-analog output used to control the analog signal on the $25 \mathrm{AFE}$

- Light-emitting diode driver outputs used to control charger status light-emitting diodes.

A custom program was written for this project to control the $25 \mathrm{AFE}$ and communicate with the bi-directional network as needed.

\subsection{GridPoint Vehicle Connectivity Modules}

The GridPoint VCMs are designed for use as a combination vehicle parameter data logger and vehicle smart grid charge unit with power meter. There are three VCMs used in this project, only the vehicle VCM is being used as the modules were designed. The other two modules have been modified slightly to take advantage of the power meter feature and the pre-established connection to the GridPoint servers. All logged information is saved on the GridPoint servers, which also serve as the platform for running the control algorithm, graphical user interface, and web application programming interface (API) described in Section 4.

The VCMs associated with the air conditioner (A/C) and fast charger measure the power and energy going into each unit. The input power to the $\mathrm{A} / \mathrm{C}$ unit is single-phase, 240-VAC power. Because the VCMs are already set up to meter single-phase alternating current, the only modification was to change the voltage-sensing circuitry to allow for the increase from 120 to 240 VAC. This VCM also is connected to a separate $\mathrm{CCC}$ board to utilize one of the relay outputs on the CCC board to control the power to the A/C. The relay is in series with the thermostat on/off control line to the $\mathrm{A} / \mathrm{C}$. This allows the $\mathrm{A} / \mathrm{C}$ unit to be turned off by the system, while still allowing the thermostat to turn the unit on based on room temperature. The CAN protocol on the CCC board and the VCM is used to indicate when the A/C unit should be turned off or set to normal operation. 
The input power into the fast charger is three-phase, 480 -VAC power. This power format presented a problem, because the VCMs are designed for use with single-phase power. To solve this problem, it was decided that a single leg of the three-phase power could be metered and custom firmware developed that assumes that the three lines are balanced. From this assumption, a simple calculation is made in the firmware that gives a good estimate of the three-phase power into the fast charger. This VCM also communicates, via CAN, with the fast charger and the L5 battery pack's battery management system. During charging, the L 5 battery management system outputs battery parameters, which are logged by the VCM. The VCM outputs charge and discharge power limits to the fast charger. These limits are adhered to by the charger within the confines of the batteries' limitations.

Each VCM sends and receives data from the GridPoint server via an Internet connection. The rate at which the VCMs send this data is specified by the design of the modules and the ability to connect to the servers.

\subsection{Site Meter}

To meter and record the power and energy into the building power panel, an ION 8600C power meter from Schneider Electric was used. The ION $8600 \mathrm{C}$ has "power and energy metering, event and $\mathrm{min} / \mathrm{max}$ logging, 32-channel historical logging, dip/swell monitoring, harmonics (to $31 \mathrm{st}$ ) and symmetrical component measurement, set points, digital and analog I/O, and Internet-enabled multi-port communications", capabilities. This multitude of functionality notwithstanding, the primary reason for the selection of this particular meter was because of its ability to connect to the GridPoint server via an Ethernet communication port and DNP 3.0 protocol. This allowed for all the parameters recorded by the meter to be accessed and logged by the control system. With this, a real-time log of the building power can be seen and used to control the power limits of the fast charger and the logic for turning off the $\mathrm{A} / \mathrm{C}$ unit.

\subsection{System Requirements}

The power flow from the transmission line to the building power panel to each end load is shown in Figure 3. With individual branch power for the fast charger and the $\mathrm{A} / \mathrm{C}$ unit, as well as the entire site power being monitored, the power flow to controlled loads (fast charger and $\mathrm{A} / \mathrm{C}$ unit) and uncontrolled loads (lights and outlets mainly; denoted as 'building loads' in the figure) can be seen. If more controlled loads were desired, the total cost would increase, as would the system complexity. This system also could be less expensive and have less complexity if the only controlled load was the fast-charge unit. These minimum system requirements would still require a site meter and a charger $\mathrm{AC}$ power meter similar to those used for this demonstration.

\section{Bi-Directional Communication Network}

\subsection{Hardware-to-Hardware Communication}

All hardware-to-hardware connections communicate via a CAN protocol. Messages are based on the GridPoint VCM message structure, with additional custom messages created for this project. Figure 4 shows the entire communication network, including each individual component.

\footnotetext{
${ }^{\mathrm{b}}$ http://www.schneider-electric.com/corporate/en/products-services/electrical-distribution/products-offer/rangepresentation.page?p_function_id=161\&p_family_id=441\&p_range_id=1462.
} 


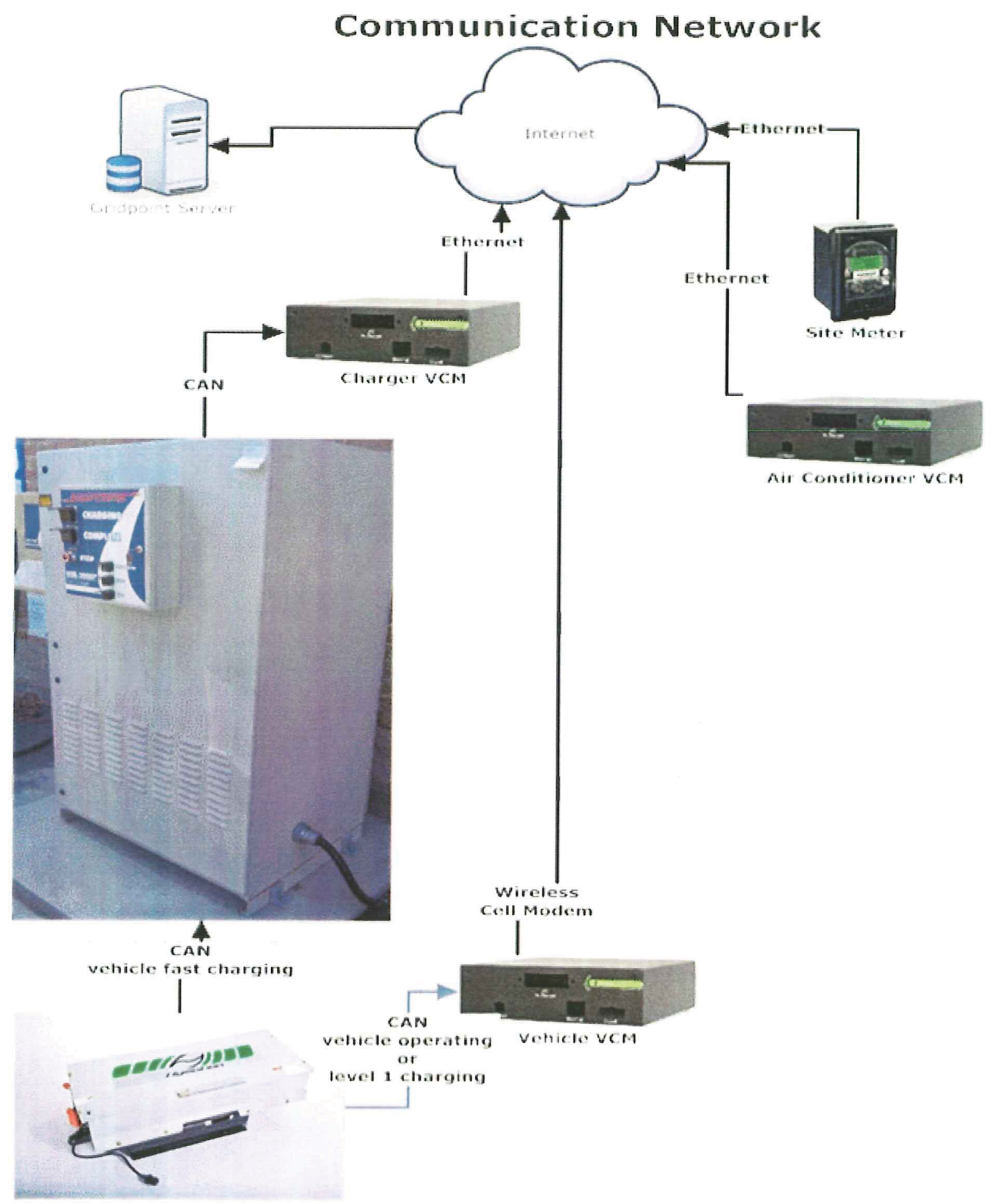

Figure 4. Communication network diagram. ${ }^{\mathrm{c}}$

${ }^{\mathrm{c}} \mathrm{A} / \mathrm{C}$ unit is not shown in picture because there are no direct communications with it. 


\subsection{Control Algorithm}

The site management control algorithm attempts to balance the metered site power so it is near the site limit set in the graphical user interface (GUI). If the site limit is set above the metered power, the algorithm will attempt to raise the actual power by increasing the charging power into the battery pack. If the site limit is below the metered power, the algorithm will attempt to lower the actual power by either discharging the battery pack or turning off the $\mathrm{A} / \mathrm{C}$ unit. Full control of the algorithm is dependent on the user-defined variables shown on the GUI.

\subsection{Graphical User Interface}

The GUI shows the real-time power values for the site meter, the charger, and the A/C unit. A screen shot of the GUI is shown in Figure 5. The GUI also shows five user-controlled fields that define how the control algorithm will operate. These five fields are defined as follows:

1. Site limit: This variable specifies the desired power use, in $\mathrm{kW}$, of the site panel. Represented as a red line on the GUI.

2. A/C priority/fast charger priority: This variable controls which resource has priority if the site limit is set to a value that does not allow both resources to operate at full power. If the $\mathrm{A} / \mathrm{C}$ priority is selected, the algorithm will try to maintain the metered power near the site limit by decreasing the charge power or discharging the battery pack. If the fast charger priority is selected, the algorithm will attempt to turn off the $\mathrm{A} / \mathrm{C}$ before decreasing charger power or discharging the battery pack.

3. Use vehicle to grid: If this variable is selected, the charger will attempt to discharge the battery pack if the metered power is below the set site limit. The power level of the discharge is determined by the difference between the actual power and the site limit, as well as the limitations of the battery pack set forth in the CCC software.

4. Disable all: This algorithm function causes an attempt to turn off all devices and keep them off. All controllable devices have inherent controls that can and will override this setting to maintain the intended applications.

5. Dead band: This setting specifies the acceptable range of variation of the site limit value versus the actual power, outside of which the algorithm will attempt to adjust the power output. For example, if the site limit is set to $5 \mathrm{~kW}$ and the dead band is set to $0.2 \mathrm{~kW}$, the algorithm will not attempt to adjust power output at any point between $4.8 \mathrm{~kW}$ and $5.2 \mathrm{~kW}$.

\subsection{Web Application Programming Interface}

There are three entities that play a part in bi-directional charging: vehicle owners, building owners, and utility companies. With this in mind, a web API was created to allow the GUI fields to be altered independent of the GUI, making it possible for other programs to be written for specific use by each end user. This could include a smart grid application that allows the vehicle owner to specify how the vehicle battery is used (i.e., by specifying the charge/discharge and state-of-charge limits). This independent program would continuously monitor and change fields based on the defined preferences. For this project, a program was designed to mimic the laboratory testing cycle and it was used to demonstrate the entire system. The general calls used by the API for this program code are shown in Appendix B. 


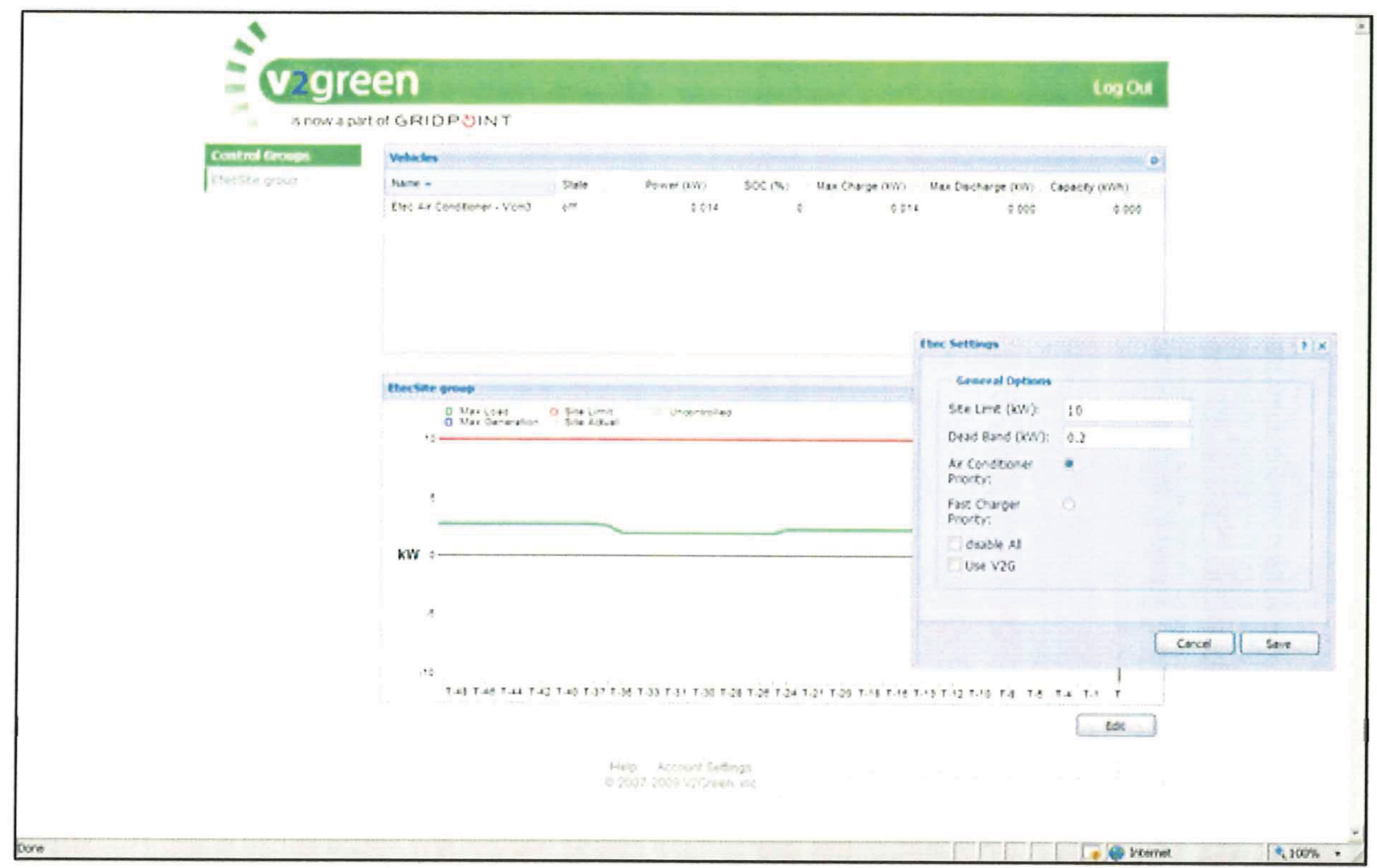

Figure 5. Graphical user interface with variable settings window.

\section{SUMMARY}

To summarize, tasks have been combined to develop a user-controllable, bi-directional vehicle charging system. The system was developed with the objective being to demonstrate the technology necessary to achieve a real-life working system. The system is currently operational and has been used to gather information to further understand the characteristics of bi-directional charging. This demonstration shows that a bi-directional vehicle charging system is possible using current commercially available technology.

\section{APPENDIXES}

Appendix A, Data Log Headers for Site Meter

Appendix B, Web Application Programming Interface Calls 


\title{
Appendix A \\ Data Log Headers for Site Meter
}

\author{
Instantaneous values: \\ ETEC_VLN_A_SCALED \\ ETEC_VA_TOTAL_SCALED \\ ETEC_VLN_B_SCALED \\ ETEC_PF_A_SCALED \\ ETEC_VLN_C_SCALED \\ ETEC_PF_B_SCALED \\ ETEC_VLN_AVG_SCALED \\ ETEC_PF_C_SCALED \\ ETEC_VLL_AB_SCALED \\ ETEC_PF_TOTAL_SCALED \\ ETEC_VLL_BC_SCALED \\ ETEC_V_UNBAL \\ ETEC_VLL_CA_SCALED \\ ETEC_I_UNBAL \\ ETEC_VLL_AVG_SCALED \\ ETEC_FREQ \\ ETEC_I_A_SCALED \\ ETEC_KW_SD_DEL_REC \\ ETEC_I_B_SCALED \\ ETEC_KVAR_SD_DEL_REC \\ ETEC_I_C_SCALED \\ ETEC_KVA_SD_DEL_REC \\ ETEC_I_AVG_SCALED \\ ETEC_KW_TOT_MX \\ ETEC_W_A_SCALED \\ ETEC_W_B_SCALED \\ //Lifetime Counters: \\ ETEC_W_C_SCALED \\ ETEC_KWH_DEL \\ ETEC_W_TOTAL_SCALED: \\ ETEC_KWH_REC \\ ETEC_VAR_A_SCALED \\ ETEC_KWH_DEL_PLUS_REC \\ ETEC_VAR_B_SCALED \\ ETEC_KWH_DEL_MINUS_REC \\ ETEC_VAR_C_SCALED \\ ETEC_KVARH_DEL \\ ETEC_VAR_TOTAL_SCALED \\ ETEC_KVARH_REC \\ ETEC_VA_A_SCALED \\ ETEC_KVARH_DEL_PLUS_REC \\ ETEC_VA_B_SCALED \\ ETEC_KVARH_DEL_MINUS_REC \\ ETEC_VA_C_SCALED \\ ETEC_KVAH_DEL_PLUS_REC
}

ETEC_NO_CONNECTIVITY: This will be set to true if there is no connectivity to the site. 


\title{
Appendix B Web Application Programming Interface Calls
}

\author{
B.1 login.sh \\ \#!/bin/bash \\ \# \\ $\# \log$ in
}

SERVER=https://sitemanagement.v2green.com

COOKIESFILE=v2greencookies.txt

USERNAME $=<$ USERNAME HERE $>$

PASSWORD $=<$ PASSWORD HERE $>$

$\mathrm{URL}=\$\{$ SERVER $\} / \operatorname{LogIn}$

wget --proxy --no-check-certificate --keep-session-cookies --save-cookies \$COOKIESFILE -O - --postdata "UserName=\$USERNAME\&Password=\$PASSWORD" $\$$ URL $>/ \mathrm{dev} / \mathrm{null}$

\section{B.2 getsettings.sh}

\#!/bin/bash

\#

\# get the settings for a particular control group

\# use an existing session to get the settings json

\# URL: https://server.v2green.com/DataServlet?dataSet=getsettings\&controlgroupid=<groupid>

. ./login.sh

CONTROLGROUPID $=1000100$

$\mathrm{URL}=\$\{$ SERVER $\} /$ DataServlet?dataSet $=$ getsettings $\mid \&$ controlgroupid $=\$\{$ CONTROLGROUPID $\}$

DATAFILE=settings.txt

wget --proxy --no-check-certificate --load-cookies \$COOKIESFILE -O \$DATAFILE \$URL 


\section{B.3 setsettings.sh}

\#!/bin/bash

\#

\# set the settings for a particular control group

\# use an existing session to set the settings json

\# URL: https://server.v2green.com/DataServlet?dataSet=getsettings\&controlgroupid=<groupid>

. .login.sh

CONTROLGROUPID $=1000100$

$\mathrm{URL}=\$\{$ SERVER $\} /$ DataServlet

SETTINGS="\{\"siteLimit|":10000,|"deadZonel":200,"noSocPrioritized":false,|"disableAll|":false,|"gener ationDisabledl":false\}"

wget --proxy --no-check-certificate --load-cookies \$COOKIESFILE -O - --post-data "dataSet=setsettings\&controlgroupid $=\$\{$ CONTROLGROUPID $\}$ \&settings $=\$\{$ SETTINGS $\}$ " \$URL > $/ \mathrm{dev} / \mathrm{null}$ 